\title{
Autonomous Demand Side Management Based on Game-Theoretic Energy Consumption Scheduling for the Future Smart Grid
}

\author{
Amir-Hamed Mohsenian-Rad, Member, IEEE, Vincent W.S. Wong, Senior Member, IEEE, Juri Jatskevich, Senior \\ Member, IEEE, Robert Schober, Fellow, IEEE, and Alberto Leon-Garcia, Fellow, IEEE
}

\begin{abstract}
Most of the existing demand side management programs focus primarily on the interactions between a utility company and its customers/users. In this paper, we present an autonomous and distributed demand side energy management system among users that takes advantage of a two-way digital communication infrastructure which is envisioned in the future smart grid. We use game theory and formulate an energy consumption scheduling game, where the players are the users and their strategies are the daily schedules of their household appliances and loads. It is assumed that the utility company can adopt adequate pricing tariffs that differentiate the energy usage in time and level. We show that for a common scenario, with a single utility company serving multiple customers, the global optimal performance in terms of minimizing the energy costs is achieved at the Nash equilibrium of the formulated energy consumption scheduling game. The proposed distributed demand side energy management strategy requires each user to simply apply its best response strategy to the current total load and tariffs in the power distribution system. The users can maintain privacy and do not need to reveal the details on their energy consumption schedules to other users. We also show that users will have the incentives to participate in the energy consumption scheduling game and subscribing to such services. Simulation results confirm that the proposed approach can reduce the peakto-average ratio of the total energy demand, the total energy costs, as well as each user's individual daily electricity charges.
\end{abstract}

Index Terms - Demand side management, distributed algorithms, energy consumption scheduling, energy pricing, game theory, market incentives, smart grid, smart meter.

\section{INTRODUCTION}

Demand side management (DSM) commonly refers to programs implemented by utility companies to control the energy consumption at the customer side of the meter [1]. These programs are employed to use the available energy more efficiently without installing new generation and transmission infrastructure. DSM programs include conservation

Manuscript was received on April 7, 2010; revised on July 5, 2010; and accepted on August 15, 2010. Paper no. TSG-00045-2010.

A. H. Mohsenian-Rad is with the Department of Electrical and Computer Engineering, Texas Tech University, Lubbock, TX 79409, USA, e-mail: hamed.mohsenian@ttu.edu. This work was completed when he was with the Department of Electrical and Computer Engineering, University of British Columbia, Vancouver, BC, Canada, V6T 1Z4 and with the Department of Electrical and Computer Engineering, University of Toronto, Toronto, ON, Canada, M5S 2E4. V. W. S. Wong, J. Jatskevich, and R. Schober are with the Department of Electrical and Computer Engineering, University of British Columbia, Vancouver, BC, Canada, V6T 1Z4, e-mails: \{vincentw, jurij, rschober\}@ece.ubc.ca. A. Leon-Garcia is with the Department of Electrical and Computer Engineering, University of Toronto, Toronto, ON, Canada, M5S 2E4, e-mail: alberto.leongarcia@utoronto.ca. and energy efficiency programs, fuel substitution programs, demand response programs, and residential or commercial load management programs [2]-[4]. Residential load management programs usually aim at one or both of the following design objectives: reducing consumption and shifting consumption [5]. The former can be achieved among users by encouraging energy-aware consumption patterns and by constructing more energy efficient buildings. However, there is also a need for practical solutions to shift the high-power household appliances to off-peak hours to reduce the peak-to-average ratio (PAR) in load demand. Appropriate load-shifting is foreseen to become even more crucial as plug-in hybrid electric vehicles (PHEVs) become popular. Most PHEVs need $0.2-0.3 \mathrm{KWh}$ of charging power for one mile of driving [6]. This will represent a significant new load on the existing distribution system. In particular, during the charging time, the PHEVs can almost double the average household load and drastically exacerbate the already high PAR. Moreover, unbalanced conditions resulting from an increasing number of PHEVs may lead to further degradation of the power quality, voltage problems, and even potential damage to utility and consumer equipment if the system is not properly reinforced [6].

One approach in residential load management is direct load control (DLC) [7]-[10]. In DLC programs, based on an agreement between the utility company and the customers, the utility or an aggregator, which is managed by the utility, can remotely control the operations and energy consumption of certain appliances in a household. For example, it may control lighting, thermal comfort equipment (i.e., heating, ventilating, and air conditioning), refrigerators, and pumps. However, when it comes to residential load control and home automation, users privacy can be a major concern and even a barrier in implementing DLC programs [11].

An alternative for DLC is smart pricing, where users are encouraged to individually and voluntarily manage their loads, e.g., by reducing their consumption at peak hours [12]-[14]. In this regard, critical-peak pricing (CPP), time-of-use pricing (ToUP), and real-time pricing (RTP) are among the popular options. For example, in RTP tariffs, the price of electricity varies at different hours of the day. The prices are usually higher during the afternoon, on hot days in the summer, and on cold days in the winter [15]. RTP programs have been adopted in some places in North America, e.g., by the Illinois Power Company in Chicago [15]. While it is usually difficult and confusing for the users to manually respond to prices that 


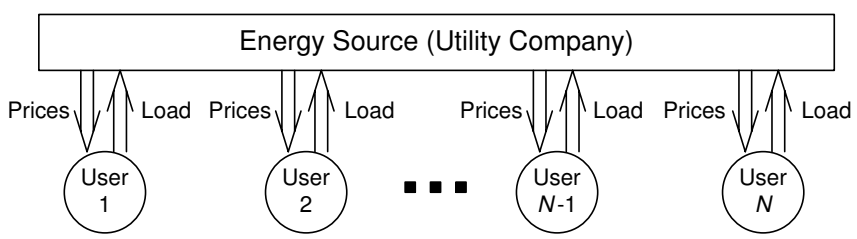

Fig. 1. A demand side management (DSM) strategy focused on individual interactions between the utility and each user.

are changing every hour [16], [17], another problem that RTP may face is load synchronization, where a large portion of load is shifted from a typical peak hour to a typical non-peak hour, without significantly reducing the PAR [18].

In most of the DSM programs that have been deployed over the past three decades (e.g., in [7]-[14]), the key focus has been on interactions between the utility company and each end user. For example, in RTP programs, each user is expected to individually respond to the time-differentiated prices by shifting its own load from the high price hours to the low price hours. Under this paradigm, each customer communicates with the energy source individually as depicted in Fig. 1. However, we argue in this paper that such an approach to the residential load control may not always achieve the best solution to the energy consumption problem. Instead, rather than focusing only on how each user behaves individually, a good DSM program should have the objective that the aggregate load satisfies some desired properties. For example, only the total load at each hour is important when it comes to solving the economic dispatching problem [19]. Also the PAR depends only on the total load demand. Therefore, while it is useful to employ aggregator units for load shaping [20], it is also important to design more efficient residential load management strategies that work by enabling interactions among users via message exchange as depicted in Fig. 2. If the users are provided with sufficient incentives, they can coordinate their usage to reduce the PAR or minimize the energy cost. Due to the recent advancements in smart grid technologies [21]-[24], the interactions between users do not have to be manual, but can be automatic through two-way digital communication.

In this paper, we propose an incentive-based energy consumption scheduling scheme for the future smart grid. We consider a scenario where a source of energy (e.g., a generator or a step-down substation transformer which is connected to the grid) is shared by several customers, each one of which is equipped with an automatic energy consumption scheduler (ECS). The ECS functionality is deployed inside the smart meters that are connected to not only the power line, but also to a communication network. The smart meters with ECS functions interact automatically by running a distributed algorithm to find the optimal energy consumption schedule for each user. The optimization objective is to minimize the energy cost in the system. As can be shown with a game-theoretic analysis [25], a simple pricing mechanism can provide the users with the incentives to cooperate. The overall system performance is improved. Each user also pays less. In other words, through an appropriate pricing scheme, the Nash equilibrium of the energy consumption game among the participating users who share the same energy source is the

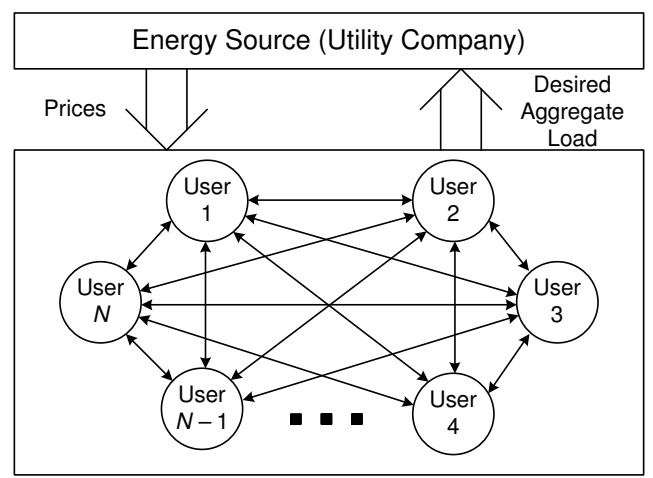

Fig. 2. A demand side management strategy for the smart grid with enabled interactions among the users/customers and the utility company.

optimal solution of a system-wide optimization problem.

The discussions and analysis in this paper extend the preliminary results in our earlier conference paper in [26] in various directions. First, here we consider not only the energy cost minimization problem but also the problem of minimizing the PAR in the total load. In this regard, we also explain the relationship between the two problems. Second, our gametheoretic analysis in this paper is more elaborate and includes new discussions on strategy-proof properties of the proposed algorithm, i.e., the ability to prevent users from cheating and misleading during their interactions with each other. Finally, the simulation results in this paper are more extensive and further include a detailed assessment of the convergence and optimality properties of our proposed algorithm, enable a better understanding of the relationship between PAR in the total load demand and the PAR in each user's individual load, and highlight the impact of changes in the number of appliances to energy consumption scheduling.

The rest of this paper is organized as follows. We introduce the system model in Section II. The PAR and energy cost minimization problems are formulated in Section III. The energy consumption games are introduced in Section IV. A distributed DSM algorithm is presented in Section V. Simulation results are given in Section VI. The paper is concluded in Section VII. All analytical proofs are summarized in Appendices A-D.

\section{SySTEM MODEL}

In this section, we provide analytical descriptions for the representation of the power system, the energy cost, and residential load control. Based on these definitions, we will formulate two design optimization problems in Section III.

\section{A. Power System}

Consider a smart power system with multiple load customers and one energy source, e.g., a generator or a step-down substation transformer connected to the electric grid. The block diagram of such a power distribution system is shown in Fig. 3. We assume that each customer is equipped with a smart meter that has an ECS capability for scheduling the household energy consumption. The smart meters are all connected to the power line coming from the energy source. They are also connected to each other and to the energy source through 


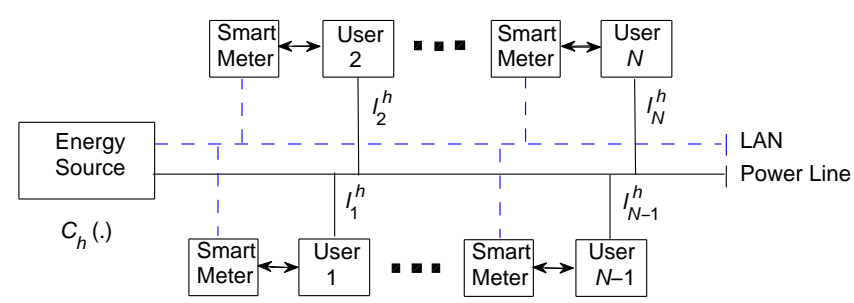

Fig. 3. Block diagram of smart grid system composed of an energy source, users, a distribution power line, and a local area communication network.

a local area network (LAN). All communications between the utility and the customers' smart meters and all message exchanges among the smart meters are done through the LAN by using appropriate communication protocols.

Throughout the paper, let $\mathcal{N}$ denote the set of users, where the number of users is $N \triangleq|\mathcal{N}|$. For each customer $n \in \mathcal{N}$, let $l_{n}^{h}$ denote the total load at hour $h \in \mathcal{H} \triangleq\{1, \ldots, H\}$, where $H=24$. Without loss of generality, we assume that time granularity is one hour. The daily load for user $n$ is denoted by $\mathbf{l}_{n} \triangleq\left[l_{n}^{1}, \ldots, l_{n}^{H}\right]$. Based on these definitions, the total load across all users at each hour of the day $h \in \mathcal{H}$ can be calculated as

$$
L_{h} \triangleq \sum_{n \in \mathcal{N}} l_{n}^{h}
$$

The daily peak and average load levels are calculated as

$$
L_{\text {peak }}=\max _{h \in \mathcal{H}} L_{h}
$$

and

$$
L_{\text {avg }}=\frac{1}{H} \sum_{h \in \mathcal{H}} L_{h},
$$

respectively. Therefore, the PAR in load demand is

$$
\mathrm{PAR}=\frac{L_{\text {peak }}}{L_{\text {avg }}}=\frac{H \max _{h \in \mathcal{H}} L_{h}}{\sum_{h \in \mathcal{H}} L_{h}} .
$$

\section{B. Energy Cost Model}

We define a cost function $C_{h}\left(L_{h}\right)$ indicating the cost of generating or distributing electricity by the energy source at each hour $h \in \mathcal{H}$. In general, the cost of the same load can be different at different times of the day. In particular, the cost can be less at night compared to the day time. In addition, we make the following assumptions throughout this paper.

Assumption 1: The cost functions are increasing. That is, for each $h \in \mathcal{H}$, the following inequality holds:

$$
C_{h}\left(\hat{L}_{h}\right)<C_{h}\left(\tilde{L}_{h}\right), \quad \forall \hat{L}_{h}<\tilde{L}_{h}
$$

From (5), energy cost increases if the total load increases.

Assumption 2: The cost functions are strictly convex. That is, for each $h \in \mathcal{H}$, any real number $\hat{L}_{h}, \tilde{L}_{h} \geq 0$, and any real number $0<\theta<1$, we have [27]

$$
C_{h}\left(\theta \hat{L}_{h}+(1-\theta) \tilde{L}_{h}\right)<\theta C_{h}\left(\hat{L}_{h}\right)+(1-\theta) C_{h}\left(\tilde{L}_{h}\right)
$$
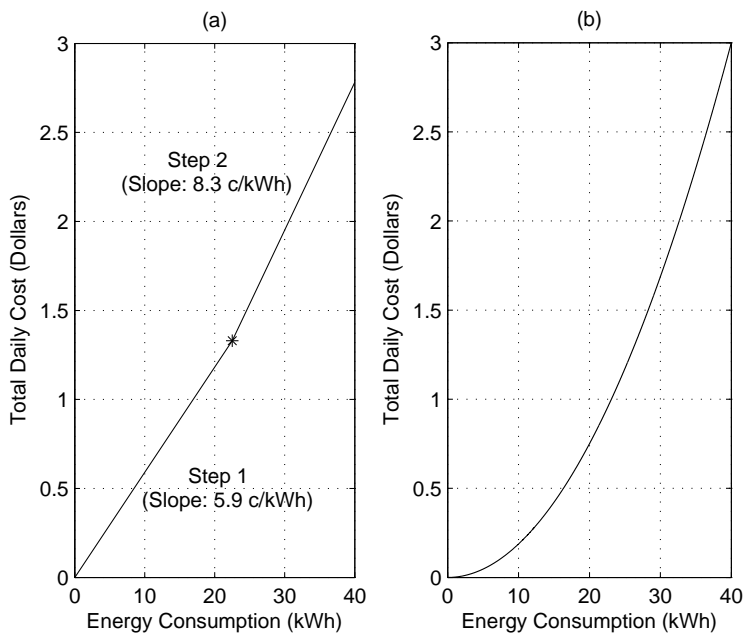

Fig. 4. Two sample convex and increasing cost functions: (a) Two-step conservation rate model used by BC Hydro [28]; (b) A quadratic cost function.

An interesting example for a class of actual energy cost functions that satisfy Assumptions 1 and 2 is the quadratic cost function for thermal generators with [19]:

$$
C_{h}\left(L_{h}\right)=a_{h} L_{h}^{2}+b_{h} L_{h}+c_{h},
$$

where $a_{h}>0$ and $b_{h}, c_{h} \geq 0$ at each hour $h \in \mathcal{H}$.

Note that the cost functions that we consider in this paper can represent either the actual energy cost as for thermal generators or simply artificial cost tariffs which are used by the utility to impose a proper load control. For example, British Columbia (BC) Hydro in Canada adopts a convex price model in form of a two-step piecewise linear function to encourage energy conservation as shown in Fig. 4(a) [28]. A smoother quadratic cost function is also shown in Fig. 4(b) which is more tractable for the purpose of optimization.

\section{Residential Load Control}

For each user $n \in \mathcal{N}$, let $\mathcal{A}_{n}$ denote the set of household appliances such as washer and dryer, refrigerator, dishwasher, air conditioner, PHEV, etc. For each appliance $a \in \mathcal{A}_{n}$, we define an energy consumption scheduling vector

$$
\mathbf{x}_{n, a} \triangleq\left[x_{n, a}^{1}, \ldots, x_{n, a}^{H}\right],
$$

where scalar $x_{n, a}^{h}$ denotes the corresponding one-hour energy consumption that is scheduled for appliance $a$ by user $n$ at hour $h$. Clearly, the total load of the $n$-th user is obtained as

$$
l_{n}^{h}=\sum_{a \in \mathcal{A}_{n}} x_{n, a}^{h}, \quad h \in \mathcal{H} .
$$

In our design, as illustrated in Fig. 5, the task of the ECS function in user $n$ 's smart meter is to determine the optimal choice of the energy consumption vector $\mathbf{x}_{n, a}$ for each appliance $a$. This will shape user $n$ 's daily load profile due to (9). Next, we identify the feasible choices of the energy consumption scheduling vectors based on users' energy needs.

For each user $n \in \mathcal{N}$ and each appliance $a \in \mathcal{A}_{n}$, we denote the pre-determined total daily energy consumption as 


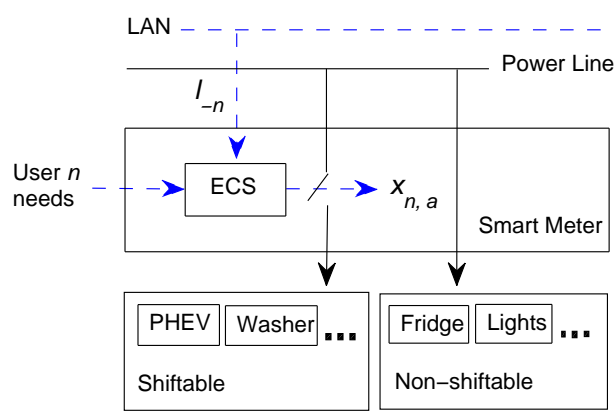

Fig. 5. The operation of the smart meter with ECS capability in our design.

$E_{n, a}$. For example, $E_{n, a}=16 \mathrm{kWh}$ for a PHEV for a 40 mile daily driving range [6]. In this paper, our designed energy consumption scheduler does not aim to change the amount of energy consumption, but instead to systematically manage and shift it, e.g., in order to reduce the PAR or minimize the energy cost. In this case, the user needs to select the beginning $\alpha_{n, a} \in$ $\mathcal{H}$ and the end $\beta_{n, a} \in \mathcal{H}$ of a time interval that appliance $a$ can be scheduled. Clearly, $\alpha_{n, a}<\beta_{n, a}$. For example, a user may select $\alpha_{n, a}=11 \mathrm{PM}$ and $\beta_{n, a}=8 \mathrm{AM}$ for its PHEV to have it ready before going to work. This imposes certain constraints on vector $\mathbf{x}_{n, a}$. In fact, the time interval for which appliance $a$ can be scheduled equals its required pre-determined daily consumption, that is

$$
\sum_{h=\alpha_{n, a}}^{\beta_{n, a}} x_{n, a}^{h}=E_{n, a}
$$

and

$$
x_{n, a}^{h}=0, \quad \forall h \in \mathcal{H} \backslash \mathcal{H}_{n, a},
$$

where $\mathcal{H}_{n, a} \triangleq\left\{\alpha_{n, a}, \ldots, \beta_{n, a}\right\}$. For each appliance, the time interval provided by the user needs to be larger than or equal to the time interval needed to finish the operation. For example, for a PHEV the normal charging time is 3 hours [6]; therefore, it is required that $\beta_{n, a}-\alpha_{n, a} \geq 3$. We also note that from (10) and (11), the total energy consumed by all appliances in the system over 24 hours is equal to the sum of the daily energy consumption of all loads/appliances. That is, we always have the following energy balance relationship:

$$
\sum_{h \in \mathcal{H}} L_{h}=\sum_{n \in \mathcal{N}} \sum_{a \in \mathcal{A}_{n}} E_{n, a} .
$$

In general, the operation of some appliances may not be time shiftable and they may have strict energy consumption scheduling constraints. For example, a refrigerator may have to be on all the time. In that case, $\alpha_{n, a}=1$ and $\beta_{n, a}=$ 24. As shown in Fig. 5, the ECS function in the smart meter essentially has no impact on the energy consumption scheduling for non-shiftable household appliances.

We define the minimum standby power level $\gamma_{n, a}^{\min }$ and the maximum power level $\gamma_{n, a}^{\max }$ for each appliance $a \in \mathcal{A}_{n}$ for each user $n \in \mathcal{N}$. Standby power refers to the electric power consumed by each appliance while it is switched off or it is in a standby mode. We assume that

$$
\gamma_{n, a}^{\min } \leq x_{n, a}^{h} \leq \gamma_{n, a}^{\max }, \quad \forall h \in \mathcal{H}_{n, a} .
$$

For notational simplicity, for each user $n$, we introduce vector $\mathbf{x}_{n}$, which is formed by stacking up energy consumption scheduling vectors $\mathbf{x}_{n, a}$ for all appliances $a \in \mathcal{A}_{n}$. In this regard, we can define a feasible energy consumption scheduling set corresponding to user $n$ as follows:

$$
\begin{aligned}
& \mathcal{X}_{n}=\left\{\mathbf{x}_{n} \mid \sum_{h=\alpha_{n, a}}^{\beta_{n, a}} x_{n, a}^{h}=E_{n, a},\right. \\
& x_{n, a}^{h}=0, \quad \forall h \in \mathcal{H} \backslash \mathcal{H}_{n, a}, \\
& \left.\gamma_{n, a}^{\min } \leq x_{n, a}^{h} \leq \gamma_{n, a}^{\max }, \quad \forall h \in \mathcal{H}_{n, a}\right\} .
\end{aligned}
$$

An energy consumption schedule calculated by the ECS unit in user $n$ 's smart meter is valid only if we have $\mathbf{x}_{n} \in \mathcal{X}_{n}$. We are now ready to formulate various energy consumption scheduling optimization problems in a smart grid.

\section{Problem Formulation}

In this section, we formulate two optimization problems based on two common design objectives in a power distribution system: PAR minimization and energy cost minimization. We show that these two problems can be related to each other depending on the choice of the energy cost function.

\section{A. Peak-to-average Ratio Minimization}

By using the expressions in (1), (9), (10), and (11) in (4), we can rewrite the PAR in terms of energy consumption scheduling vectors $\mathbf{x}_{1}, \ldots, \mathbf{x}_{N}$ as

$$
\frac{H \max _{h \in \mathcal{H}}\left(\sum_{n \in \mathcal{N}} \sum_{a \in \mathcal{A}_{n}} x_{n, a}^{h}\right)}{\sum_{n \in \mathcal{N}} \sum_{a \in \mathcal{A}_{n}} E_{n, a}} .
$$

In general, a low PAR is preferred [19]. Therefore, given complete knowledge about the users' needs and the smart grid depicted in Fig. 3, an efficient energy consumption scheduling can be characterized as the solution to the following problem:

$$
\underset{\mathbf{x}_{n} \in \mathcal{X}_{n}, \forall n \in \mathcal{N}}{\operatorname{minimize}_{n \in \mathcal{N}^{\prime}}} \frac{H \max _{h \in \mathcal{H}}\left(\sum_{n \in \mathcal{N}} \sum_{a \in \mathcal{A}_{n}} x_{n, a}^{h}\right)}{\sum_{n \in \mathcal{N}} \sum_{a \in \mathcal{A}_{n}} E_{n, a}} .
$$

Next, we note that since $H$ and $\sum_{n \in \mathcal{N}} \sum_{a \in \mathcal{A}_{n}} E_{n, a}$ are fixed as far as the optimization variables $\mathbf{x}_{1}, \ldots, \mathbf{x}_{N}$ are concerned, they can be removed from the objective function and we can rewrite problem (16) as the following equivalent problem:

$$
\underset{\mathbf{x}_{n} \in \mathcal{X}_{n}, \forall}{\operatorname{minimize}} \max _{h \in \mathcal{H}}\left(\sum_{n \in \mathcal{N}} \sum_{a \in \mathcal{A}_{n}} x_{n, a}^{h}\right) .
$$

However, problem (17) is still difficult to solve in its current form due to the max term in the objective function. This can be resolved by introducing a new auxiliary variable $\Gamma$ and rewriting problem (17) in equivalent form as

$$
\begin{array}{ll}
\underset{\Gamma, \mathbf{x}_{n} \in \mathcal{X}_{n}, \forall n \in \mathcal{N}}{\operatorname{minimize}} & \Gamma \\
\text { subject to } & \Gamma \geq \sum_{n \in \mathcal{N}} \sum_{a \in \mathcal{A}_{n}} x_{n, a}^{h}, \quad \forall h \in \mathcal{H} .
\end{array}
$$


Problem (18) is a linear program. It can be solved in a centralized fashion by using either the simplex method or the interior point method (IPM) [27], [29]. We also note that problem (18) may have more than one optimal solution. That is, the same minimum PAR in the total load demand can be achieved through different energy consumption schedules.

\section{B. Energy Cost Minimization}

An efficient energy consumption scheduling can also be formulated in terms of minimizing the energy costs to all users, which can be expressed as the following optimization problem:

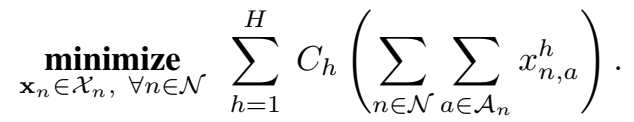

Optimization problem (19) is convex and can be solved in a centralized fashion using convex programming techniques such as IPM [27], [29]. Since the cost functions are assumed to be strictly convex, minimization problem (19) always has a unique solution, given the choices of the cost functions [27]. This is one of the differences between the energy cost minimization problem and the PAR minimization problem. Recall that the latter could have multiple optimal-solutions.

\section{EnERgy CONSUMPTION GAME}

Although the optimization problems defined in the previous section can be solved in a centralized fashion to obtain an optimal solution for a given configuration of the users and their energy schedules, it is more advantageous to define a solution approach that can be implemented and updated autonomously to accommodate the changes within the system. For these reasons, we are interested in solving problem (19) in a distributed way at the level of the smart meter using it's ECS functionality, and with a minimum amount of information exchanges among the smart meters and the energy source. In particular, the goal is to let each smart meter with ECS functionality schedule the energy consumption of the household according to the individual needs of the users. It is also important to make sure that the users have an incentive to actually use the ECS features and to follow the schedules they determine. We focus on the energy cost minimization problem. Nevertheless, we will see in Section VI that by achieving the minimum cost, we also achieve a low PAR in the total load demand.

\section{A. Pricing and Billing Tariffs}

For each user $n \in \mathcal{N}$, let $b_{n}$ denote the daily billing amount in dollars to be charged to user $n$ by the utility at the end of each day. The prices should reflect the users' total daily energy consumption and relate it to the total energy cost of the system. It is reasonable to assume that

$$
\sum_{n \in \mathcal{N}} b_{n} \geq \sum_{h=1}^{H} C_{h}\left(\sum_{n \in \mathcal{N}} l_{n}^{h}\right)
$$

where the left hand side in (20) denotes the total daily charge to the users and the right hand side denotes the total daily cost. For notational simplicity we define

$$
\kappa \triangleq \frac{\sum_{n \in \mathcal{N}} b_{n}}{\sum_{h=1}^{H} C_{h}\left(\sum_{n \in \mathcal{N}} l_{n}^{h}\right)} \geq 1
$$

If $\kappa=1$, then the billing system is budget-balanced and the utility company charges the users only with the same amount that generating/providing energy costs for the utility. On the other hand, if $\kappa>1$, then the difference between the total charges to the users and the total energy cost would indicate the profit made by the utility company. We further assume that

$$
\frac{b_{n}}{b_{m}}=\frac{\sum_{h=1}^{H} l_{n}^{h}}{\sum_{h=1}^{H} l_{m}^{h}}, \quad \forall n, m \in \mathcal{N} .
$$

That is, users are charged proportional to their total daily energy consumption. For example, if user $n$ consumes twice as much energy as user $m$, then he/she will be charged twice as much as user $m$. The exact amount of the charge depends on the cost of energy at each hour of the day, which itself results from a strictly convex function $C(\cdot)$ such as the one shown in Fig. 4. While the assumption in (22) helps in keeping our analysis tractable by directly relating every user's total payment to the total energy cost in the system, it is also consistent with the existing residential metering models. We are now ready to introduce an efficient energy pricing model which satisfies both assumptions (20) and (22).

After summing up the two sides of the equality in (22) across all users $m \in \mathcal{N}$, for each $n \in \mathcal{N}$, we have

$$
\begin{aligned}
\sum_{m \in \mathcal{N}} b_{m} & =\sum_{m \in \mathcal{N}}\left(b_{n} \frac{\sum_{h=1}^{H} l_{m}^{h}}{\sum_{h=1}^{H} l_{n}^{h}}\right) \\
& =b_{n} \frac{\sum_{m \in \mathcal{N}} \sum_{h=1}^{H} l_{m}^{h}}{\sum_{h=1}^{H} l_{n}^{h}} .
\end{aligned}
$$

Together from (9), (10), (21), and (23) and after reordering the terms we can show that for each user $n \in \mathcal{N}$ we have

$$
\begin{aligned}
b_{n} & =\frac{\sum_{h=1}^{H} l_{n}^{h}}{\sum_{m \in \mathcal{N}} \sum_{h=1}^{H} l_{m}^{h}}\left(\sum_{m \in \mathcal{N}} b_{m}\right) \\
& =\frac{\kappa \sum_{h=1}^{H} l_{n}^{h}}{\sum_{m \in \mathcal{N}} \sum_{h=1}^{H} l_{m}^{h}}\left(\sum_{h=1}^{H} C_{h}\left(\sum_{m \in \mathcal{N}} l_{m}^{h}\right)\right) \\
& =\Omega_{n} \sum_{h=1}^{H} C_{h}\left(\sum_{m \in \mathcal{N}} \sum_{a \in \mathcal{A}_{m}} x_{m, a}^{h}\right),
\end{aligned}
$$

where

$$
\Omega_{n} \triangleq \frac{\kappa \sum_{a \in \mathcal{A}_{n}} E_{n, a}}{\sum_{m \in \mathcal{N}} \sum_{a \in \mathcal{A}_{m}} E_{m, a}} .
$$

Next, we study the behavior of the users when they are charged according to (24) by using techniques from game theory.

\section{B. Game Model}

From (24), the charge on each user depends on how he and all other users schedule their consumptions. This naturally leads to the following game among users:

Game 1 (Energy Consumption Game Among Users):

- Players: Registered users in set $\mathcal{N}$.

- Strategies: Each user $n \in \mathcal{N}$ selects its energy consumption scheduling vector $\mathbf{x}_{n}$ to maximize its payoff. 
- Payoffs: $P_{n}\left(\mathbf{x}_{n} ; \mathbf{x}_{-n}\right)$ for each user $n \in \mathcal{N}$, where

$$
\begin{aligned}
P_{n}\left(\mathbf{x}_{n} ; \mathbf{x}_{-n}\right) & =-b_{n} \\
& =-\Omega_{n} \times\left(\sum_{h=1}^{H} C_{h}\left(\sum_{m \in \mathcal{N}} \sum_{a \in \mathcal{A}_{m}} x_{m, a}^{h}\right)\right) .
\end{aligned}
$$

Here, $\mathbf{x}_{-n} \triangleq\left[\mathbf{x}_{1}, \ldots, \mathbf{x}_{n-1}, \mathbf{x}_{n+1}, \ldots, \mathbf{x}_{N}\right]$ denotes the vector containing the energy consumption schedules of all users other than end user $n$.

Based on the definitions of the payoffs and strategies in Game 1, the users try to select their energy consumption schedule to minimize their payments to the utility company.

Theorem 1: Suppose Assumptions 1 and 2 hold. The Nash equilibrium of Game 1 always exists and is unique.

The proof of Theorem 1 is given in Appendix A. Note that Nash equilibrium is a solution concept in game theory that characterizes how the players play a game [25]. The energy consumption scheduling variables $\left(\mathbf{x}_{n}^{*}, \forall n \in \mathcal{N}\right)$ form a Nash equilibrium for Game 1 if and only if we have

$$
P_{n}\left(\mathbf{x}_{n}^{*} ; \mathbf{x}_{-n}^{*}\right) \geq P_{n}\left(\mathbf{x}_{n} ; \mathbf{x}_{-n}^{*}\right), \quad \forall n \in \mathcal{N}, \mathbf{x}_{n} \geq 0 .
$$

If the energy consumption game is at its unique Nash equilibrium, then no user would benefit by deviating from schedule $\left(\mathbf{x}_{n}^{*}, \forall n \in \mathcal{N}\right)$. Next, we show the following result regarding the performance at Nash equilibrium of Game 1.

Theorem 2: The Nash equilibrium of Game 1 is the optimal solution of energy cost minimization problem (19).

The proof of Theorem 2 is given in Appendix B. From Theorems 1 and 2, as long as the cost functions $C_{h}(\cdot)$ are increasing and strietly convex for each $h \in \mathcal{H}$ and also the price model satisfies the requirements (20) and (22), the users have an incentive to cooperate with each other to reduce their own payments by solving problem (19).

\section{Distributed Algorithm}

From the results in Section IV, the users would be willing to cooperate and allow their ECS units to schedule their household energy consumption to pay less. In particular, we showed that the unique Nash equilibrium of the energy consumption game among the users is the same as the global optimal solution of the energy consumption scheduling problem (19). In this section, we provide an algorithm to be implemented in each ECS unit to reach the Nash equilibrium of Game 1 and achieve the optimal system performance. We prove the convergence and optimality properties of the proposed algorithm. We also show that it is strategy-proof and users will not benefit from misleading each other by providing inaccurate information about their usage during their interactions.

\section{A. Principle of the Algorithm}

Consider any user $n \in \mathcal{N}$. Given $\mathbf{x}_{-n}$ and assuming that all other users fix their energy consumption schedule according to $\mathbf{x}_{-n}$, user $n$ 's best response can be determined by solving the following local optimization problem:

$$
\underset{\mathbf{x}_{n} \in \mathcal{X}_{n}}{\operatorname{maximize}} P_{n}\left(\mathbf{x}_{n} ; \mathbf{x}_{-n}\right) .
$$

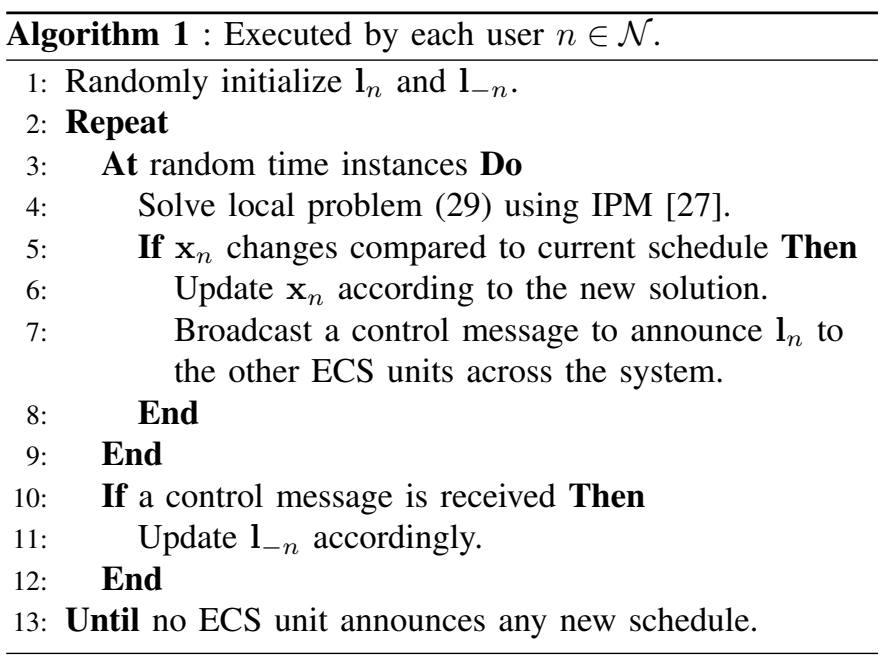

Notice that we refer to optimization problem (27) as a local problem for user $n$ because the only optimization variable is user $n$ 's energy consumption scheduling vector $\mathbf{x}_{n}$. Since $\Omega_{n}$ is fixed and does not depend on the choice of $\mathbf{x}_{n}$, the maximization in (27) can be replaced by

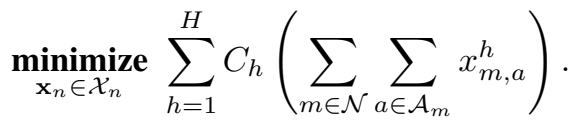

After reordering the terms, we can also rewrite (28) as

$$
\underset{\mathbf{x}_{n} \in \mathcal{X}_{n}}{\operatorname{minimiz}} \sum_{h=1}^{H} C_{h}\left(\sum_{a \in \mathcal{A}_{n}} x_{n, a}^{h}+\sum_{m \in \mathcal{N} \backslash\{n\}} l_{m}^{h}\right) .
$$

We notice that problems (29) and (19) have the same objective functions. However, problem (29) has only local variables for user $n$. Moreover, problem (29) is convex and can be solved by IPM [27]. User $n$ can solve problem (29) as long as it knows the cost functions $C_{h}$ for all $h \in \mathcal{H}$ as well as $\mathbf{l}_{-n} \triangleq\left[\mathbf{l}_{1}, \ldots, \mathbf{l}_{n-1}, \mathbf{l}_{n+1}, \ldots, \mathbf{l}_{N}\right]$, i.e., the vector containing the scheduled daily energy consumption for all other users. These observations motivate us to propose Algorithm 1 to solve problem (19) in a distributed fashion.

Next, we explain how the proposed algorithm works. In Line 1 , each user $n \in \mathcal{N}$ starts with some random initial conditions. That is, each user $n$ assumes a random vector $\mathbf{l}_{m}$ for any $m \in \mathcal{N} \backslash\{n\}$. This assumption is required since at the beginning, user $n$ has no prior information about other users. Then, the loop in Lines 2 to 13 is executed until the algorithm converges ${ }^{1}$. Within this loop, each ECS individually solves its own version of local optimization problem (29) using IPM in Line 4. That is, each user simply plays its best response as discussed in Section V-A. The new schedule is announced to the other users through broadcasting a control message. In fact, the message exchange between the users in the general framework in Fig. 2 are implemented in Algorithm 1 in form of each user $n$ broadcasting $l_{n}$ over the LAN. Note that users do not reveal the details about the energy consumption of their own appliances due to privacy concerns. They only announce their total hourly usage, which is collected at the

\footnotetext{
${ }^{1}$ We will discuss the convergence property of Algorithm 1 in Theorem 3.
} 
energy source for billing purposes anyways. In this setting, each user also updates its local memory whenever it receives a control message from other users in Line 11.

The proposed DSM strategy in this section has key differences with the existing DSM programs in the literature. First, unlike DLC, here each user has control over the operation of its own appliances. Therefore, user privacy is not a concern. Moreover, the users simply follow what is best for them in order to decide on their own consumption schedules while they select the best-response in the energy consumption game. Different from DLC and RTP which are based on the framework in Fig. 1, our design is based on the new setting in Fig. 2 and incorporates the interactions among users.

\section{B. Convergence and Optimality}

In this section, we prove the convergence and optimality properties of the proposed distributed algorithm. The cornerstone of our assessment is the following theorem.

Theorem 3: If the updates of the individual energy consumption scheduling vectors are asynchronous among the users, i.e., no two users $n, m \in \mathcal{N}$ update their energy consumption scheduling vectors $\mathbf{x}_{n}$ and $\mathbf{x}_{m}$ at the same time, then starting from any randomly selected initial conditions, Algorithm 1 converges to its fixed point, i.e., to the Nash equilibrium of the energy consumption game.

The proof of Theorem 3 is given in Appendix C. Theorem 3 provides a sufficient condition to guarantee convergence. This condition only requires the users to update their energy consumption scheduling vectors sequentially. For example, this can be achieved if the energy source can determine the timing when each user should update its energy consumption. In that case, instead of Line 3 in Algorithm 1, user $n$ would execute Lines 4 to 7 only if it receives a command from the energy source telling him/her that it is user $n$ 's turn to update its energy consumption scheduling vectors. Other turntaking scenarios can also be used to coordinate the energy consumption scheduling updates among the users.

Together, from Theorems 2 and 3, starting from any initial point, Algorithm 1 automatically converges to the global optimal solution of energy cost minimization problem (19). We notice that if all users' energy consumption needs remain unchanged within the next 24 hours, then Algorithm 1 becomes a day-ahead energy consumption scheduling design. However, if the energy consumption needs for the users change frequently, then Algorithm 1 will converge to the new optimal energy consumption schedules in a more real-time fashion (cf. [18]).

\section{Strategy-proof Property}

In this section, we would like to answer this question: Is it beneficial for a user or a group of users to cheat and announce an incorrect energy consumption schedule to the other users? That is, does it help user $n$, in terms of increasing its daily payoff at a fixed point of Algorithm 1, to be untruthful and set $\mathbf{l}_{n} \neq\left(\sum_{a \in \mathcal{A}_{n}} x_{n, a}^{h}\right)$ ? Of course, it is possible to have the energy source supervise and monitor all message exchanges among users such that the users' truthfulness can be enforced.
But it is still interesting to see if Algorithm 1 itself enforces truthfulness with no extra supervisory effort from the utility.

Theorem 4: When the users are running Algorithm 1, no user or group of users would benefit from being untruthful. That is, each user $n \in \mathcal{N}$ will end up having a higher electricity payment on its daily bill if he announces its daily energy consumption schedule $l_{n}$ incorrectly.

The proof of Theorem 4 is given in Appendix D. From Theorem 4 we can assure that all users release their daily energy consumption schedule accurately. This includes the case when a user believes that all other users in the system are being truthful as well. This implies that an energy consumption game is not tempting for cheating and is fundamentally different from some well-known games such as the prisoner's dilemma game [25, p. 110] where the users do cheat if they think that other players are truthful. It is worth clarifying that the underlying cause for Algorithm 1 to be automatically strategy-proof is that in our billing model, we have directly related every user's payoff to the total energy cost in the system. In fact, the global and individual cost minimizations are closely related in our model. Therefore, any behavior by a user or a group of users which results in deviating from the optimal system performance will also harm the cheating user or the group of cheating users in terms of individual payments, deterring users from any malicious behavior.

\section{Simulation Results}

In this section, we present simulation results and assess the performance of our proposed algorithm. In our considered benchmark smart grid system there are $N=10$ customer/users that subscribe to the ECS services. For the purpose of study, each user is selected to have between 10 to 20 appliances with non-shiftable operation, i.e., with strict energy consumption scheduling constraints. Such appliances may include refrigerator-freezer (daily usage: $1.32 \mathrm{kWh}$ ), electric stove (daily usage: $1.89 \mathrm{kWh}$ for self-cleaning and $2.01 \mathrm{kWh}$ for regular), lighting (daily usage for 10 standard bulbs: 1.00 $\mathrm{kWh}$ ), heating (daily usage: $7.1 \mathrm{kWh}$ ) [30]. Moreover, each user is selected to also have between 10 to 20 appliances with shiftable operation, i.e., with soft energy consumption scheduling constraints. Recall that the smart meter with ECS capability may schedule only the appliances with soft energy consumption scheduling constraints. Such appliances may include dishwasher (daily usage: $1.44 \mathrm{kWh}$ ), washing machine (daily usage: $1.49 \mathrm{kWh}$ for energy-star, $1.94 \mathrm{kWh}$ for regular), clothes dryer (daily usage: $2.50 \mathrm{kWh}$ ), and PHEV (daily usage: $9.9 \mathrm{kWh}$ ). [6], [30]. In our simulation model, we assume that each user has a randomly selected combination of the considered shiftable and non-shiftable loads to be used at different times of the day by taking into account that the load demand is higher in the evening and lower during the night. For example, we assumed that when PHEVs become popular as widely predicted, it is reasonable to assume that most users ( 4 out of 5 users in our setting) have electric cars to be charged some time between the afternoon hours on each day and the early morning hours on the next day. The energy cost function is assumed to be quadratic as in (7). For simplicity we assume 
that $b_{h}=c_{h}=0$ for all $h \in \mathcal{H}$. We also have $a_{h}=0.3$ cents at day-time hours, i.e., from 8:00 in the morning to 12:00 at night and $a_{h}=0.2$ cents during the night, i.e., from 12:00 at night to 8:00 AM the day after. The power system is assumed budgetbalanced, i.e., $\kappa=1$ (see (21)). The timing Algorithm 1 works based on a round-robin scenario which is coordinated by the energy source. In this scenario, at each user's turn it will start its local computation to update its own energy consumption schedule according to Line 4 in Algorithm 1. Then it will inform the energy source who will allocate turn to another randomly selected user and this procedure continues until the algorithm converges. In this setting, the energy source makes sure that every user takes a turn once-in-while.

\section{A. Performance Comparison}

The simulation results on total scheduled energy consumptions and the energy cost for a single scenario are shown in Figs. 6 and 7, without and with the deployment of the ECS function in the smart meters, respectively. For the case without ECS deployment, each appliance $a \in \mathcal{A}_{n}$ for each user $n \in \mathcal{N}$ is assumed to start operation right at the beginning of the time interval $\left[\alpha_{n, a}, \beta_{n, a}\right]$ and at its typical power level. For the case with ECS deployment, the timing and the power level for the operation of each household appliance is determined by Algorithm 1. By comparing the results in Figs. 6 and 7, we can see that when the ECS functions are not used/implemented, the PAR is 2.1 and the energy cost is $\$ 44.77$. At the same time, when the ECS feature is enabled, the PAR reduces to 1.8 (i.e., $17 \%$ less) and the energy cost reduces to $\$ 37.90$ (i.e., $18 \%$ less). In fact, in the latter case, there is a more evenly distributed load across different hours of the day. Note that each user consumes the same amount of energy in the two cases, but it simply schedules its consumption more efficiently in the case that the ECS units are used. On the other hand, the trends of the resulting total energy cost while Algorithm 1 proceeds along its distrbuted iterations are shown in Fig. 8. We can see that as the users run Algorithm 1, the energy cost monotonically decreases until the algorithm converges after 22 iterations only, i.e., around 2 iterations per user on average.

\section{B. User Payment}

While the proposed distributed DSM strategy leads to less total energy cost and lower PAR in the aggregate load demand, it is also beneficial for each individual end user. To see this, the daily payments for all users are shown in Fig. 9. Here, the simulation setting is the same as the one in Section VIA. We can see that all users would pay significantly less to the utility company when the ECS is enabled in the smart meter. Therefore, the users would be willing to participate in the proposed automatic demand side management system.

Another interesting aspect is shown in Fig. 10. In this figure, we have plotted the PAR in each user's load and compared it with the PAR in the aggregate load across all users. Here, for each user $n \in \mathcal{N}$, the individual PAR is calculated as

$$
\mathrm{PAR}_{n} \triangleq \frac{H \max _{h} l_{n}^{h}}{\sum_{h=1}^{H} l_{n}^{h}}
$$
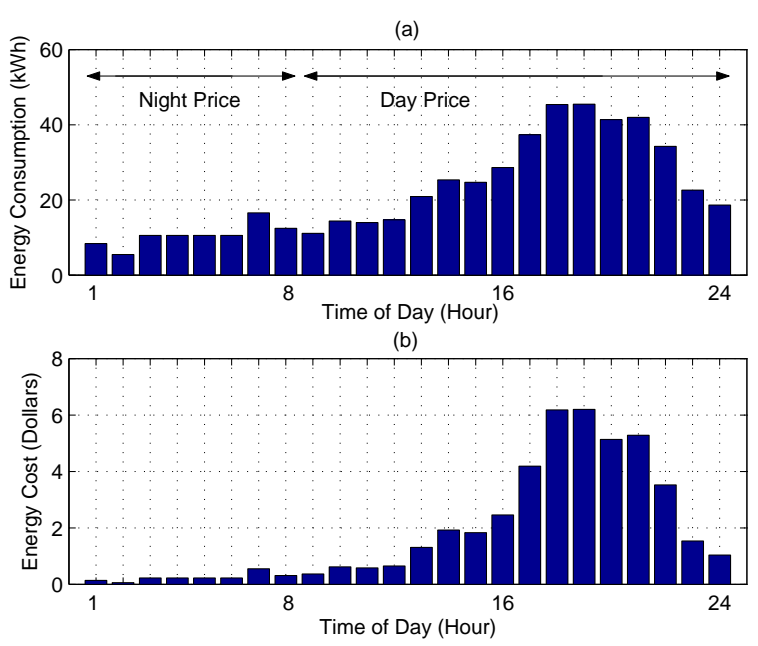

Fig. 6. Scheduled energy consumption and corresponding cost when ECS units are not used. In this case, PAR is 2.1 and the total daily cost is $\$ 44.77$.

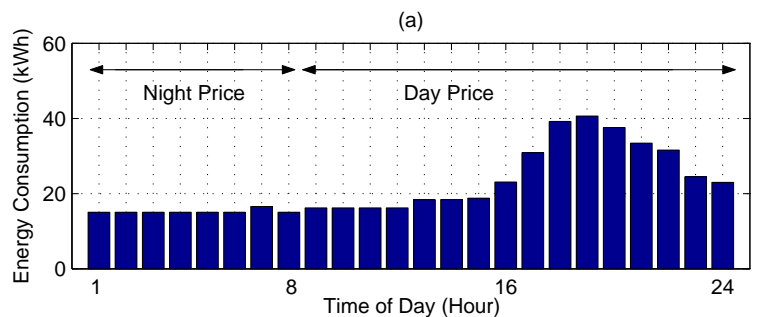

(b)

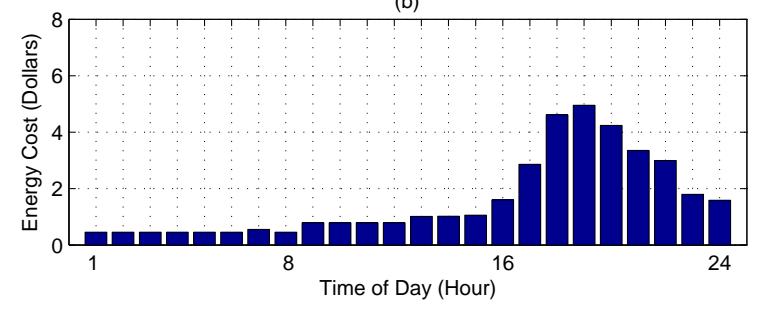

Fig. 7. Scheduled energy consumption and corresponding cost when ECS units are deployed. In this case, PAR is 1.8 and the total daily cost is $\$ 37.90$.

We can see in Fig. 10 that the PAR in the aggregate load is significantly less than the PAR in each user's individual load. In fact, for some users, such as user 7 in our example scenario, the load is quite unbalanced and the PAR is around 4.5. This confirms our discussions in Section I that the utility company may not necessarily need all users to individually balance their load, as opposed to the design objective in realtime pricing tariffs which expect each individual end user to shift its consumption from peak hours to off-peak hours.

\section{Optimal PAR Reduction}

Recall from Section III that in order to achieve the minimum PAR in the total load demand, we can schedule energy consumption according to the optimal solution of problem (18). However, our proposed distributed algorithm in this paper only aims at minimizing the energy cost, i.e., solving problem (19). In this section, we show that by solving problem (19), Algorithm 1 results in PAR values which are very close 


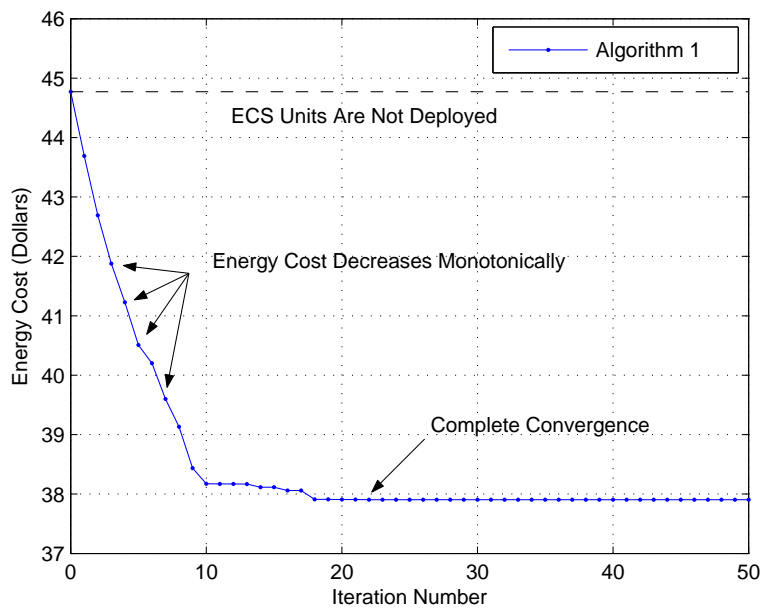

Fig. 8. Trend of resulting energy cost along the iterations of Algorithm 1. We can see that the proposed distributed algorithm converges quickly. Steady state is reached after only 22 iterations when the energy cost is minimized.

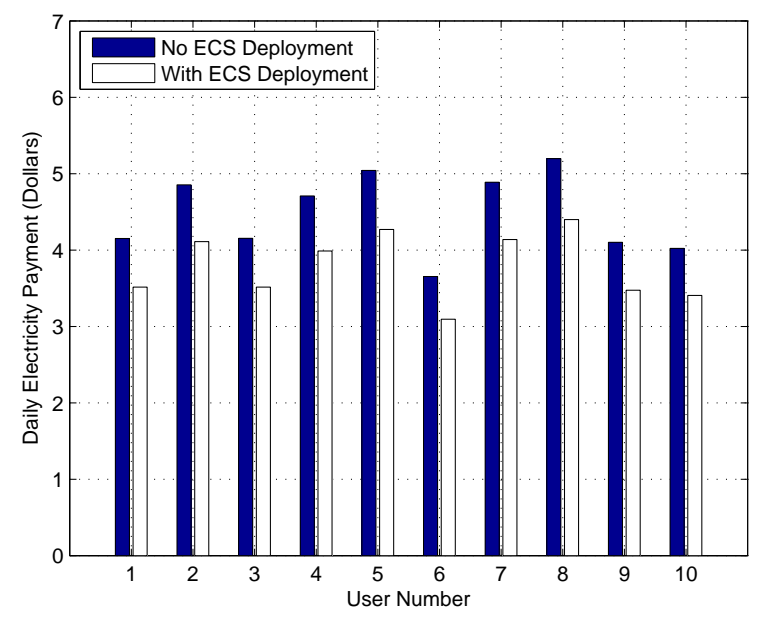

Fig. 9. Daily charges for each subscriber without and with ECS deployment.

to the PAR values obtained by solving problem (18). In addition, Algorithm 1 significantly reduces the energy cost. Corresponding simulation results are shown in Fig. 11. Here, we simulated 50 different scenarios to have a more accurate comparison. From the results in Fig. 11(a), we can see that the PARs achieved by solving the PAR minimization problem (18) and the energy cost minimization problem (19) are almost the same. In fact, in 32 out of 50 scenarios, the two PAR values are identical. In the remaining 18 scenarios, the solution of the PAR minimization problem results in strictly lower PAR, e.g., as in scenario number 17. However, even in these cases, the improvement is minor. On average, the PAR reduces from 1.8325 to 1.8315 (i.e., only $0.05 \%$ improvement). On the other hand, from Fig. 11(b), we can see that Algorithm 1 significantly outperforms the optimal solution of the PAR minimization problem in terms of reducing the energy cost. We notice that while the average energy cost when no ECS unit is deployed is as high as $\$ 51.83$, the average energy cost at the optimal solution of the PAR minimization problem reduces

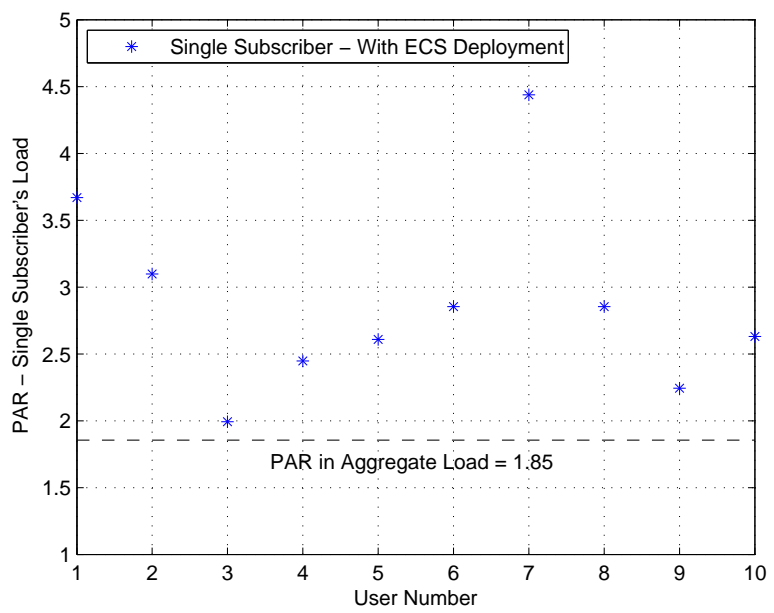

Fig. 10. PAR in each end user's individual daily load and comparison with the PAR in the aggregate load across all end users.

to $\$ 48.04$. However, the average energy cost at the optimal solution of the energy cost minimization problem obtained by running Algorithm 1 is only $\$ 41.65$. Therefore, we can conclude that Algorithm 1, which is designed for energy cost minimization, also efficiently reduces the PAR in the aggregate load. Moreover, it can significantly reduce the energy cost.

\section{Impact of Amount of Shiftable Load}

For the simulation scenarios so far, we have assumed that around half of the residential load is shiftable while the other half is not shiftable. Clearly, the ECS units are expected to have a more significant impact if more appliances have shiftable operation. To better see this, we have plotted the PAR in the aggregate load demand when the percentage of shiftable load varies from $10 \%$ to $90 \%$ in Fig. 12. In this regard, we can see that if 9 out of 10 appliances have shiftable operation, then on average, the PAR in the aggregate load can be reduced down to only 1.35 , indicating almost a flat load.

\section{CONCLUSions AND Future Work}

In this paper, we proposed an optimal, autonomous, and distributed incentive-based energy consumption scheduling algorithm in order to minimize the cost of energy and also to balance the total residential load when multiple users share a common energy source. Unlike most of the previous DSM strategies that focus solely on the interactions between the utility company and each user, the basis of our design are the interactions among the users. Our proposed distributed algorithm requires only some limited message exchanges between users when each of them tries to maximize its own benefits in a game-theoretic setting. In order to encourage users to behave in a desired way (i.e., to minimize the energy cost) we proposed a smart pricing tariff such that the interactions among the users automatically lead to an optimal aggregate load profile at the equilibrium of an energy consumption scheduling game. Simulation results confirm that the proposed distributed 


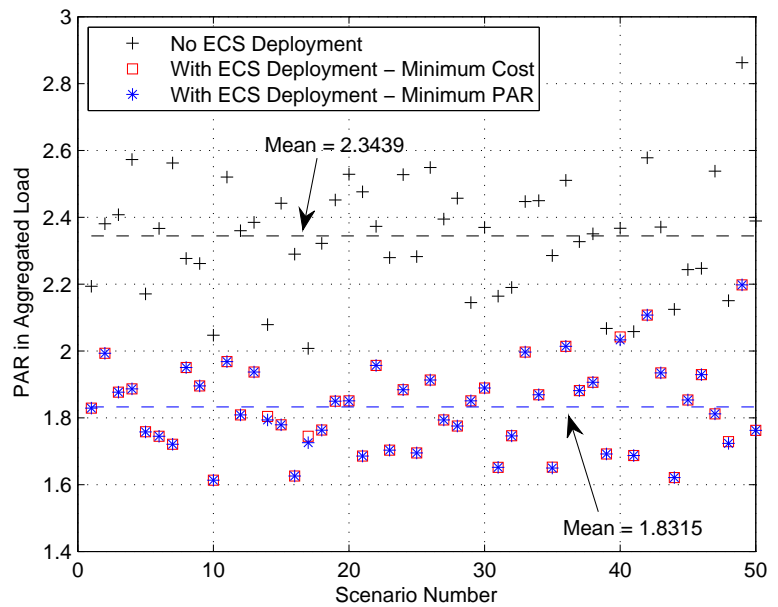

(a) Minimizing the PAR in the aggregate load.

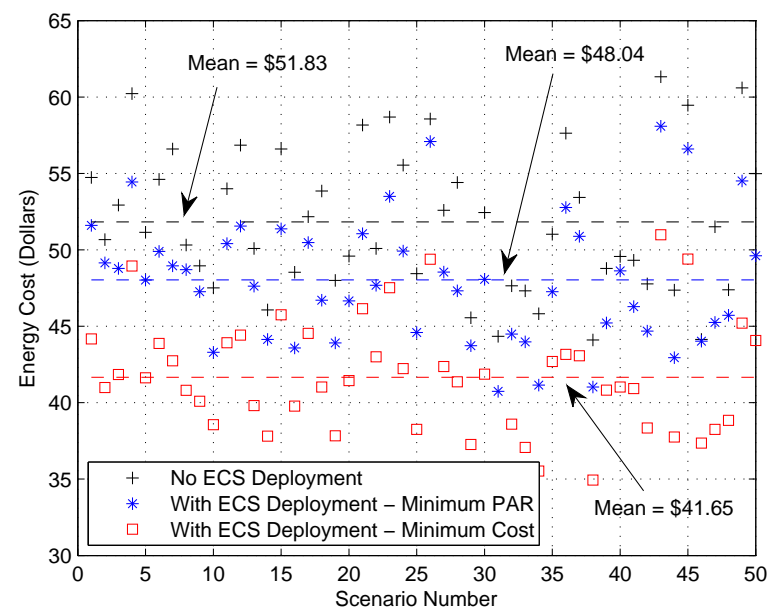

(b) Minimizing the energy cost.

Fig. 11. Comparison between the optimal solution of the PAR minimization problem (18), the optimal solution of the energy cost minimization problem (19), and the case with no ECS function deployment in smart meters.

demand side management strategy can reduce the PAR, the energy cost, and each user's daily electricity charges.

The results in this paper can be extended in several directions. First, the proposed distributed DSM strategty can be modified to address the case when there are multiple energy sources in the system. In that case, the users need to determine not only the total amount of their energy consumption at each hour of the day, but also the portion of the total energy that they need to obtain from each available energy source. Second, it is interesting to extend our design to address both shifting and reducing energy consumption. This can be done by introducing new energy cost functions which depend on not only the energy consumption at each hour, but also the total daily energy consumption. In this regard, the linear billing model in (22) in Section IV-A can be extended to more general non-linear models. Third, one may relax the convexity assumption on the choices of the energy cost functions to cover a wider range of energy cost models. Of course, this will introduce optimization problems which are more difficult

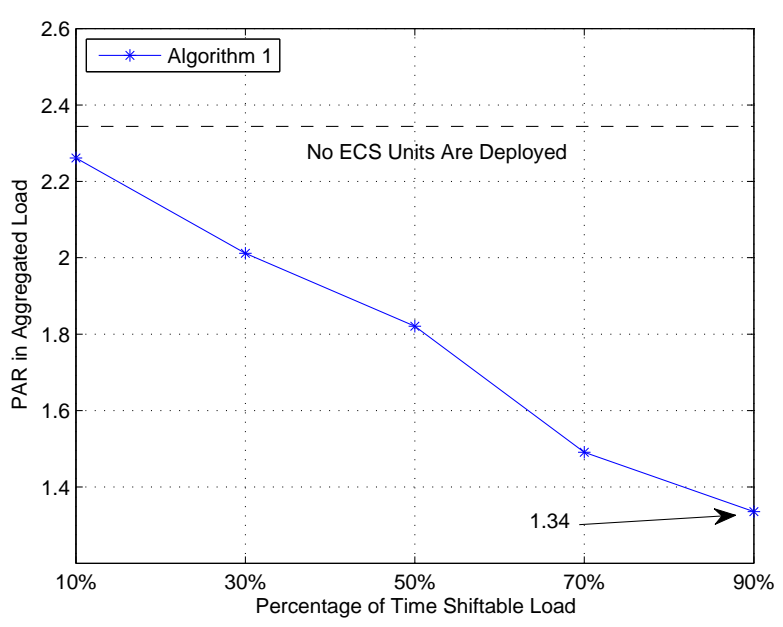

Fig. 12. The PAR in the aggregate load when the percentage of shiftable load varies from $10 \%$ to $90 \%$. In a scenario when most of the residential load is shiftable, the aggregate load can be flat resulting in a very low PAR.

to solve. Fourth, our system model can be extended to a scenario where users can store energy at certain hours, e.g., in their PHEV batteries during the night. They can then sell the energy back to the grid at peak hours. This can be done by allowing our energy consumption scheduling variables to take negative values for appliances that have energy storage capability, where a negative value for these variables indicates providing rather than consuming energy. Finally, while our analysis focused only on residential load control, similar techniques can be used to better shape the aggregate profile of commercial load in an industrial region.

\section{APPENDIX}

\section{A. Proof of Theorem 1}

We first notice that since $C_{h}(\cdot)$ is strictly convex for each $h \in \mathcal{H}$, the payoff function $P_{n}\left(\mathbf{x}_{n} ; \mathbf{x}_{-n}\right)$ is strictly concave with respect to $\mathbf{x}_{n}$. Therefore, Game 1 is a strictly concave $N$ person game. In this case, the existence of a Nash equilibrium directly results from [31, Theorem 1]. Moreover, the Nash equilibrium is unique due to [31, Theorem 3$]$.

\section{B. Proof of Theorem 2}

We first show that the global optimal solution of problem (19) forms a Nash equilibrium for Game 1. For notational simplicity, let $\mathbf{x}_{1}^{\star}, \ldots, \mathbf{x}_{N}^{\star}$ denote the optimal solution of problem (19). We also define

$$
C^{\star} \triangleq \sum_{h=1}^{H} C_{h}\left(\sum_{m \in \mathcal{N}} \sum_{a \in \mathcal{A}_{m}} x_{m, a}^{h \star}\right) .
$$

By definition of optimality, for each subscriber $n \in \mathcal{N}$ and for any arbitrary $\mathbf{x}_{n} \geq 0$, we have

$$
C^{\star} \leq \sum_{h=1}^{H} C_{h}\left(\sum_{m \in \mathcal{N} \backslash\{n\}} \sum_{a \in \mathcal{A}_{m}} x_{m, a}^{h \star}+\sum_{a \in \mathcal{A}_{n}} x_{n, a}^{h}\right) .
$$


After multiplying both sides in (32) by $-\Omega_{n}$ it becomes

$$
P_{n}\left(\mathbf{x}_{n}^{\star} ; \mathbf{x}_{-n}^{\star}\right) \geq P_{n}\left(\mathbf{x}_{n} ; \mathbf{x}_{-n}^{\star}\right), \quad \forall \mathbf{x}_{n} \geq 0 .
$$

Comparing (33) and (26), we can conclude that the optimal solution $\mathbf{x}_{1}^{\star}, \ldots, \mathbf{x}_{N}^{\star}$ forms a Nash equilibrium for Game 1. However, from Theorem 1, Game 1 has a unique Nash equilibrium. Thus, the optimal solution of problem (19) is equivalemt to the Nash equilibrium of Game 1.

\section{Proof of Theorem 3}

Recall that playing the best response for each user $n \in$ $\mathcal{N}$ would be equivalent to solving optimization problem (29). Therefore, if users play the best responses sequentially through running Algorithm 1 in an asynchronous fashion, the energy cost in the system either decreases or remains unchanged every time a user updates its energy consumption schedule. Since the energy cost is bounded below (e.g., the energy cost is always non-negative), the convergence to some fixed point is evident. On the other hand, at the fixed point of Algorithm 1, no user can improve its payoff by deviating from the fixed point when playing its best response. This directly indicates that the fixed point is the Nash equilibrium of Game 1 among the users.

\section{Proof of Theorem 4}

Let $\overline{\mathbf{x}}_{1}, \ldots, \overline{\mathbf{x}}_{N}$ denote the Nash equilibrium of Game 1 when a non-empty set of users $\mathcal{M} \subseteq \mathcal{N}$ are untruthful, while all other users $\mathcal{N} \backslash \mathcal{M}$ are truthful. Also let $\mathbf{x}_{1}^{\star}, \ldots, \mathbf{x}_{N}^{\star}$ denote the optimal solution of problem (19). Recall from Theorem 2 that $\mathbf{x}_{1}^{\star}, \ldots, \mathbf{x}_{N}^{\star}$ is also the Nash equilibrium of Game 1 when all users are truthful. For each user $n \in \mathcal{M}$ to benefit from being untruthful, it is required that we have

$$
P_{n}\left(\overline{\mathbf{x}}_{n} ; \overline{\mathbf{x}}_{-n}\right) \geq P_{n}\left(\mathbf{x}_{n}^{\star} ; \mathbf{x}_{-n}^{\star}\right) .
$$

By dividing both sides in (34) by $-\Omega_{n}$, we should have

$$
\begin{aligned}
& \sum_{h=1}^{H} C_{h}\left(\sum_{m \in \mathcal{N}} \sum_{a \in \mathcal{A}_{m}} \bar{x}_{m, a}^{h}\right) \\
& \leq \sum_{h=1}^{H} C_{h}\left(\sum_{m \in \mathcal{N}} \sum_{a \in \mathcal{A}_{m}} x_{m, a}^{\star h}\right) .
\end{aligned}
$$

However, this contradicts the fact that $\mathbf{x}_{1}^{\star}, \ldots, \mathbf{x}_{N}^{\star}$ is the optimal solution of problem (19). Therefore, user $n$ does not benefit from announcing inaccurate information with respect to its daily energy consumption schedule in Line 7 of Algorithm 1. In fact, since every user's individual payoff is nothing but the total energy cost times a negative constant $-\Omega_{n}$, for each user or a group of users, the only way to increase the payoff at Nash equilibrium is to reduce the total energy cost. Therefore, any behavior, such as being untruthful, which leads to increasing the energy cost from its optimal/minimum value would harm the cheating users or the group of cheating users as well as every other user in the system.

\section{ACKNOWLEDGEMENT}

The authors would like to thank the support from the Natural Sciences and Engineering Research Council (NSERC) of Canada and the Canada Research Chair program.

\section{REFERENCES}

[1] G. M. Masters, Renewable and Efficient Electric Power Systems. Hoboken, NJ: Wiley, 2004.

[2] B. Ramanathan and V. Vittal, "A framework for evaluation of advanced direct load control with minimum disruption," IEEE Trans. on Power Systems, vol. 23, no. 4, pp. 1681-1688, Nov. 2008.

[3] C. W. Gellings and J. H. Chamberlin, Demand Side Management: Concepts and Methods, 2nd ed. PennWell Books, 1993.

[4] M. A. A. Pedrasa, T. D. Spooner, and I. F. MacGill, "Scheduling of demand side resources using binary particle swarm optimization," IEEE Trans. on Power Systems, vol. 24, no. 3, pp. 1173-1181, Aug. 2009.

[5] Energy Conservation Committee Report and Recommendations, Reducing Electricity Consumption in Houses. Ontario Home Builders' Association, May 2006.

[6] A. Ipakchi and F. Albuyeh, "Grid of the future," IEEE Power and Energy Magazine, pp. 52-62, Mar. 2009.

[7] N. Ruiz, I. Cobelo, and J. Oyarzabal, "A direct load control model for virtual power plant management," IEEE Trans. on Power Systems, vol. 24, no. 2, pp. 959-966, May 2009.

[8] A. Gomes, C. H. Antunes, and A. G. Martins, "A multiple objective approach to direct load control using an interactive evolutionary algorithm," IEEE Trans. on Power Systems, vol. 22, no. 3, pp. 1004-1011, Aug. 2007.

[9] D. D. Weers and M. A. Shamsedin, "Testing a new direct load control power line communication system," IEEE Trans. on Power Delivery, vol. 2, no. 3, pp. 657-660, July 1987.

[10] C. M. Chu, T. L. Jong, and Y. W. Huang, "A direct load control of air-conditioning loads with thermal comfort control," in Proc. of IEEE PES General Meeting, San Francisco, CA, June 2005.

[11] OpenHAN Task Force of the UtilityAMI Working Group, Home Area Network System Requirements Specification, Aug. 2008.

[12] K. Herter, "Residential implementation of critical-peak pricing of electricity," Energy Policy, vol. 35, pp. 2121-2130, Apr. 2007.

[13] C. Triki and A. Violi, "Dynamic pricing of electricty in retail markets," Quarterly Journal of Operations Research, vol. 7, no. 1, pp. 21-36, Mar. 2009.

[14] P. Centolella, "The integration of price responsive demand into regional transmission organization (RTO) wholesale power markets and system operations (article in press)," Energy, An International Journal, vol. 35 , no. 4, pp. 1568-1574, Apr. 2010.

[15] H. Allcott, "Real Time Pricing and Electricity Markets," Working Paper, Harvard University, Feb. 2009.

[16] Quantum Consulting Inc. and Summit Blue Consulting, LLC Working Group 2 Measurement and Evaluation Committee and Southern California Edison Company, Demand Response Program Evaluation - Final Report, Apr. 2005.

[17] M. Ann-Piette, G. Ghatikar, S. Kiliccote, D. Watson, E. Koch, and D. Hennage, "Design and operation of an open, interoperable automated demand response infrastructure for commercial buildings," J. of Computing and Information Sci. in Engineering, vol. 9, pp. 1-9, June 2009.

[18] A. H. Mohsenian-Rad and A. Leon-Garcia, "Optimal residential load control with price prediction in real-time electricity pricing environments," IEEE Trans. on Smart Grid (accepted), May 2010.

[19] A. J. Wood and B. F. Wollenberg, Power Generation, Operation, and Control. Wiley-Interscience, 1996.

[20] "Comverge Demand Response Aggregatos," http://www.comverge.com/demandmanager/, 2010.

[21] U.S. Department of Energy, The Smart Grid: An Introduction, 2009.

[22] A. Vojdani, "Smart integration," IEEE Power and Energy Magazine, vol. 6, no. 6, pp. 72-79, Nov. 2008.

[23] L. H. Tsoukalas and R. Gao, "From smart grids to an energy internet: Assumptions, architectures, and requirements," in Proc. of the Third International Conference on Electric Utility Deregulation and Restructuring and Power Technologies, Nanjing, China, Apr. 2008.

[24] M. Amin and B. F. Wollenberg, "Toward a smart grid: power delivery for the 21 st century," IEEE Power and Energy Magazine, vol. 4, no. 6 , pp. 34-41, Nov. 2006.

[25] D. Fudenberg and J. Tirole, Game Theory. The MIT Press, 1991.

[26] A. H. Mohsenian-Rad, V. W. S. Wong, J. Jatskevich, and R. Schober, "Optimal and autonomous incentive-based energy consumption scheduling algorithm for smart grid," in Proc. of IEEE PES Conference on Innovative Smart Grid Technologies, Gaithersburg, MD, Jan. 2010.

[27] S. Boyd and L. Vandenberghe, Convex Optimization. Cambridge University Press, 2004. 
[28] "British Columbia Hydro Conservation Electricity Rates," Available Online at https://www.bchydro.com/youraccount/content/residential inclining_block.jsp, 2009.

[29] D. Bertsimas and J. N. Tsitsiklis, Introduction to Linear Optimization. Athena Scientific, 1997

[30] Office of Energy Efficiency, Natural Resources Canada, Energy Consumption of Household Appliances Shipped in Canada, Dec. 2005.

[31] J. B. Rosen, "Existence and uniqueness of equilibrium points for concave n-person games," Econometrica, vol. 33, pp. 347-351, 1965.

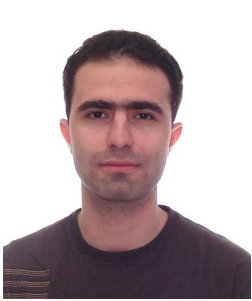

Amir-Hamed Mohsenian-Rad (S04-M09) received masters degree in Electrical Engineering from Sharif University of Technology in 2004 and Ph.D. degree in Electrical and Computer Engineering from The University of British Columbia (UBC) in 2008. Currently, he is an Assistant Professor at the Department of Electrical and Computer Engineering at Texas Tech University. Dr. Mohsenian-Rad is the recipient of the UBC Graduate Fellowship, Pacific Century Graduate Scholarship, and the Natural Sciences and Engineering Research Council of Canada (NSERC) Post-doctoral Fellowship. He is an Associate Editor of the International Journal of Electronics and Communication and serves as a Technical Program Committee member in various conferences, including IEEE Globecom, ICC, and CCNC. His research interests include design, optimization, and gametheoretic analysis of communication networks and smart power systems.

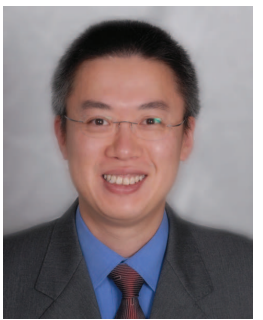

Vincent W.S. Wong (SM'07) received the B.Sc. degree from the University of Manitoba, Winnipeg, MB, Canada, in 1994, the M.A.Sc. degree from the University of Waterloo, Waterloo, ON, Canada, in 1996, and the Ph.D. degree from the University of British Columbia (UBC), Vancouver, BC, Canada, in 2000. From 2000 to 2001, he worked as a systems engineer at PMC-Sierra Inc. He joined the Department of Electrical and Computer Engineering at UBC in 2002 and is currently an Associate Professor. His research interests are in resource and mobility management for wireless mesh networks, wireless sensor networks, and heterogeneous wireless networks. Dr. Wong is an Associate Editor of the IEEE Transactions on Vehicular Technology and an Editor of KICS/IEEE Journal of Communications and Networks. He serves as TPC member in various conferences, including IEEE Infocom, ICC, and Globecom. He is a senior member of the IEEE and a member of the ACM.

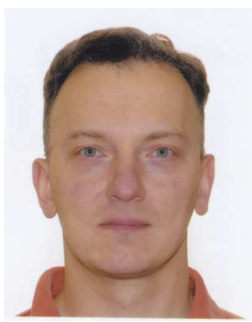

Juri Jatskevich (M99, SM07) (M99, SM07) received the M.S.E.E. and the Ph.D. degrees in Electrical Engineering from Purdue University, West Lafayette IN, USA, in 1997 and 1999, respectively. Since 2002, he has been a faculty member at the University of British Columbia, Vancouver, Canada, where he is now an Associate Professor of Electrical and Computer Engineering. Dr. Jatskevich is presently a Chair of IEEE CAS Power Systems and Power Electronic Circuits Technical Committee, Editor of IEEE Transactions on Energy Conversion, Editor of IEEE Power Engineering Letters, and Associate Editor of IEEE Power Electronics. He is also chairing the IEEE Task Force on Dynamic Average Modeling, under Working Group on Modeling and Analysis of System Transients Using Digital Programs. His research interests include smart energy grids, power electronic systems, electrical machines and drives, modeling and simulation of electromagnetic transients.

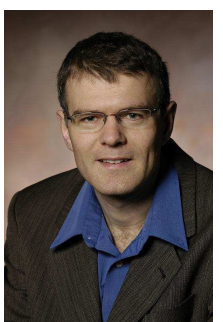

Robert Schober (M'01, SM'08, F'10) was born in Neuendettelsau, Germany, in 1971. He received the Diplom (Univ.) and the Ph.D. degrees in electrical engineering from the University of ErlangenNuermberg in 1997 and 2000, respectively. From May 2001 to April 2002 he was a Postdoctoral Fellow at the University of Toronto, Canada, sponsored by the German Academic Exchange Service (DAAD). Since May 2002 he has been with the University of British Columbia (UBC), Vancouver, Canada, where he is now a Full Professor and Canada Research Chair (Tier II) in Wireless Communications. His research interests fall into the broad areas of Communication Theory, Wireless Communications, and Statistical Signal Processing.

Dr. Schober received the 2002 Heinz MaierLeibnitz Award of the German Science Foundation (DFG), the 2004 Innovations Award of the Vodafone Foundation for Research in Mobile Communications, the 2006 UBC Killam Research Prize, the 2007 Wilhelm Friedrich Bessel Research Award of the Alexander von Humboldt Foundation, and the 2008 Charles McDowell Award for Excellence in Research from UBC. In addition, he received best paper awards from the German Information Technology Society (ITG), the European Association for Signal, Speech and Image Processing (EURASIP), IEEE ICUWB 2006, the International Zurich Seminar on Broadband Communications, and European Wireless 2000. Dr. Schober is also the Area Editor for Modulation and Signal Design for the IEEE Transactions on Communications.

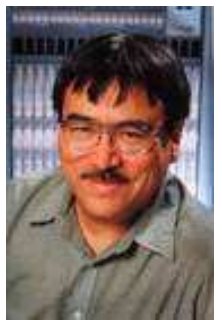

Alberto Leon-Garcia (F99) received the B.S., M.S., and $\mathrm{Ph} . \mathrm{D}$. degrees in electrical engineering from the University of Southern California, in 1973, 1974, and 1976, respectively. Currently, he is a Professor in Electrical and Computer Engineering at the University of Toronto. He is a Fellow of the IEEE "For contributions to multiplexing and switching of integrated services traffic". He is also a Fellow of the Engineering Institute of Canada. He has received the 2006 Thomas Eadie Medal from the Royal Society of Canada and the 2010 IEEE Canada A. G. L. McNaughton Gold Medal for his contributions to the area of communications He holds a Canada Research Chair in Autonomic Service Architecture. From 1999 to 2002, he was founder and CTO of AcceLight Networks in Ottawa which developed an all-optical fabric multi-terabit, multiservice core switch. He holds several patents and has published extensively in the areas of switch architecture and traffic management. Professor LeonGarcia's research interests are focused on application-oriented networking and autonomic resources management with a focus on enabling pervasive smart infrastructure. His research team is currently developing a network testbed that will enable at-scale experimentation in new network protocols and distributed applications. Professor Leon-Garcia is recognized as an innovator in networking education. In 1986, he led the development of the University of Toronto - Northern Telecom Network Engineering Program. He also led in 1997 the development of the Master of Engineering in Telecommunications program, and the communications and networking options in the undergraduate computer engineering program. He is the author of the leading textbooks: Probability and Random Processes for Electrical Engineering, and Communication Networks: Fundamental Concepts and Key Architecture. 